Measurement of the total collisional ionization rates of $\mathrm{Ne} \mathrm{VI,} \mathrm{VII} \mathrm{and} \mathrm{VIII}$

This content has been downloaded from IOPscience. Please scroll down to see the full text. 1977 J. Phys. B: At. Mol. Phys. 10187

(http://iopscience.iop.org/0022-3700/10/2/008)

View the table of contents for this issue, or go to the journal homepage for more

Download details:

IP Address: 130.237.29.138

This content was downloaded on 08/12/2015 at 21:58

Please note that terms and conditions apply. 


\title{
Measurement of the total collisional ionization rates of Ne VI, VII and VIII'
}

\author{
Larry A Jones, Elisabeth Källne and David B Thomson \\ Los Alamos Scientific Laboratory of the University of California, Los Alamos. \\ New Mexico 87544, USA
}

Received 17 May 1976, in final form 13 September 1976

\begin{abstract}
The total collisional ionization rates of neon VI, vII and viI have been measured in a time-evolving theta-pinch plasma. The method used was to calculate the expected emission from our plasma using the measured time- and space-dependent electron density $\left(10^{16}-10^{17} \mathrm{~cm}^{-3}\right)$ and temperature $(120-400 \mathrm{eV})$. The semi-empirical ionization rates of Kunze and Lotz were used in our calculation and were adjusted, by multiplying with a constant, so that the predicted photon emission matched the observed line emission. The multiplicative factors, with estimated errors, required for best fit are: for neon VI, $0.15 \pm 0.1$; for neon VII, $0.4 \pm 0.2$; and for neon VIII, $0.7 \pm 0.25$. Also, a comparison is made between the semi-empirical rates used in this work and the recently calculated rates of Summers. These recently calculated rates were in good agreement with our experiment for neon VII and VIII. For neon VI, however, the departure from our experimental result was even farther than for the semi-empirical rate.
\end{abstract}

\section{Introduction}

Accurately known values for the ionization rates of highly stripped heavy ions are necessary to predict the energy loss due to radiation from thermonuclear plasmas. This energy loss can control many factors in reactor design, one of which is the power input requirement during the heating stage. In this paper we will describe an experiment in which we measured the total ionization rates of Ne VI, VII and VIII. Neon was chosen as the heavy element to be studied because: (i) it is relatively easy to add to a theta-pinch plasma, (ii) there are none of the sweep-up problems encountered with heavier elements in a theta pinch (Datla et al 1972) and (iii) some work has already been done on Ne vI, VII and viII for a comparison (Kunze 1971a, b, Kunze and Johnston 1971, Johnston and Kunze 1971).

The method we have chosen (Kunze 1972) to measure the ionization rates of highly stripped atoms is to observe the intensity versus time of a strong line emitted by each ionic species from our theta-pinch plasma. We also must measure the value and radial distribution of the electron density and temperature as a function of time. We then use the time and space evolution of the density and temperature, together with rate equations to calculate the time-dependent emission to be expected from our plasma, using assumed values for the ionization and recombination rates. The ionization rates are then adjusted until predicted emission versus time agrees with

+ Work done under the auspices of the US ERDA. 
the observed emission. Of course this method yields values of the ionization rates which are model-dependent, i.e. depend upon the values chosen for the other rates. However, the ionization rates are probably the least accurately known quantities in the rate equations and so values obtained by this method, although having uncertainties, represent a significant improvement over the present knowledge of these rates.

\section{Experimental}

To produce the plasma in this experiment we used a theta pinch (Thomson et al 1976) with a single-turn coil, internal diameter $8.2 \mathrm{~cm}$ by $25 \mathrm{~cm}$ long, driven by a $60 \mathrm{~kJ}, 60 \mathrm{kV}$ capacitor bank. The discharge tube was a quartz tube with an inside diameter of $6.5 \mathrm{~cm}$. The filler gas consisted of $98 \%$ helium and $2 \%$ neon, and flowed continuously through the discharge tube to maintain a pressure of $20 \mathrm{mTorr}$. After each shot the tube was pumped out to a pressure of less than $10^{-4}$ Torr and then allowed to refill. The gas was partially preionized by inducing a glow discharge axially down the tube from a $15 \mathrm{kV}, 15 \mathrm{~mA} \mathrm{AC}$ transformer. About $20 \mu$ s before the $60 \mathrm{~kJ}, 60 \mathrm{kV}$ main bank was discharged, a $0.7 \mu \mathrm{F}, 60 \mathrm{kV}$ fast-ringing capacitor was discharged through the coil to produce further preionization. The main bank itself has a quarter cycle rise time of about $2 \mu \mathrm{s}$, at which time it is crowbarred, producing a field decay time of approximately $100 \mu \mathrm{s}$. A schematic diagram of the highvoltage circuit is shown in figure 1 . At current maximum the field reached inside the coil is $45 \mathrm{kG}$.

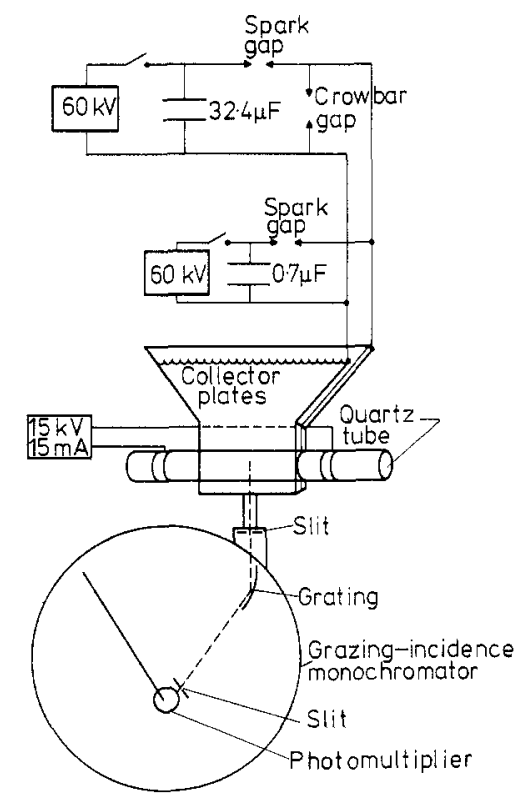

Figure 1. A schematic diagram of the theta pinch used in this experiment. Also shown is the $2.2 \mathrm{~m}$ grazing-incidence monochromator and its orientation. 
J. Phys. B: Atom. Molec. Phys., Vol. 10, No. 2. (C) 1977-L A Jones et al
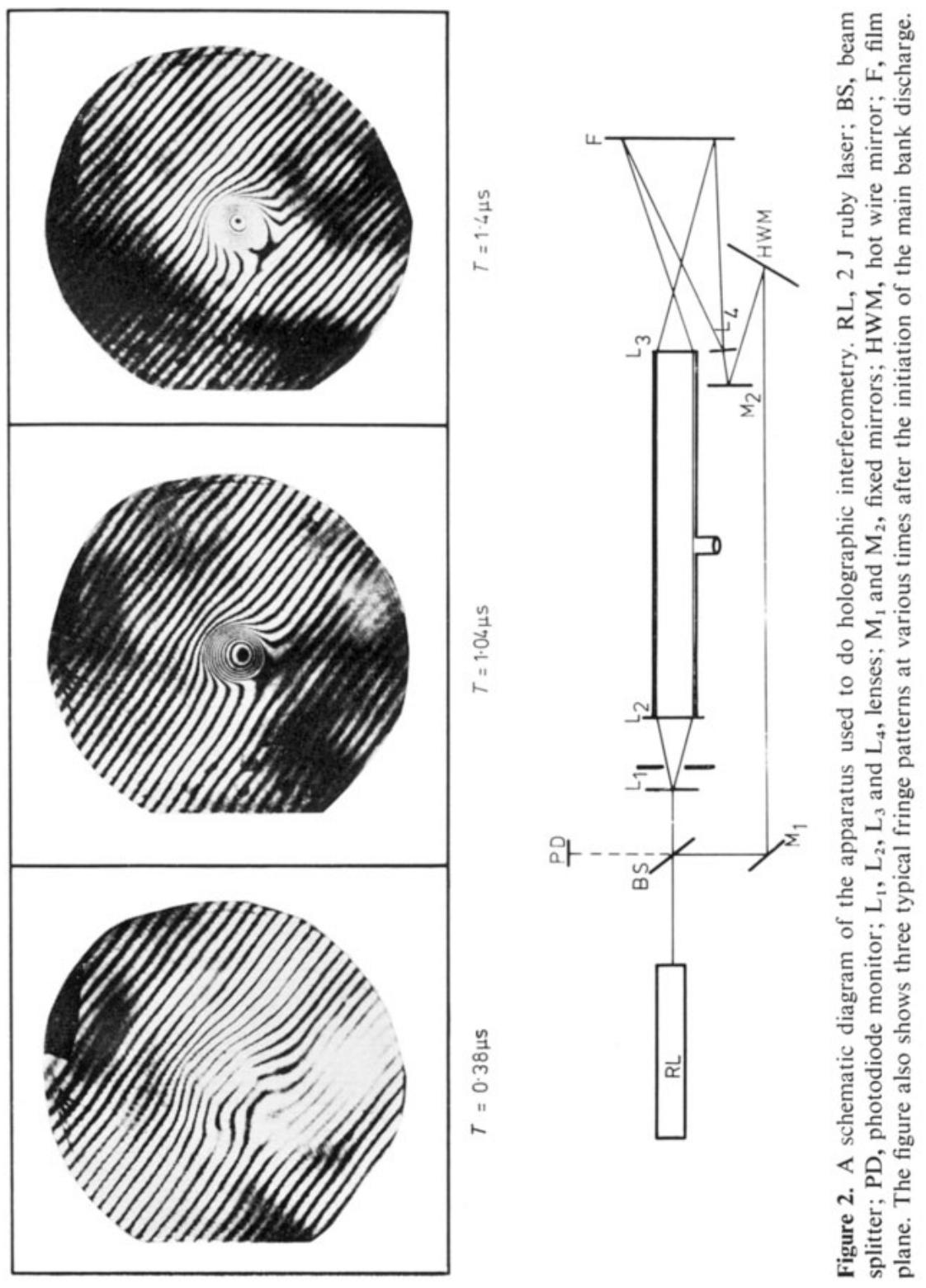
At the low pressures of filler gas used ( $20 \mathrm{mTorr}$ ), it was difficult to produce the plasma without having a linear discharge to increase the preionization. We found that this linear discharge was not necessary for preionization for the first few discharges after the discharge tube was pumped down from atmospheric pressure. This behaviour we interpret as being due to easily ionized impurity atoms clinging to the discharge-tube walls and being removed after a few discharges. At higher pressures of filler gas the ringing preionization was always sufficient for a reproducible plasma.

The vacuum ultraviolet radiation emitted by the strong lines of $\mathrm{Ne} \mathrm{V}$-vIII was dispersed by a $2.2 \mathrm{~m}$ grazing-incidence monochromator. The radiation was detected with a p-terphenyl coated EMI 6256B photomultiplier tube. The plasma was observed side-on through a $13 \mathrm{~mm}$ diameter port, which gave a vacuum line of sight to the plasma column and allowed viewing of the entire plasma diameter. A schematic diagram of the apparatus is shown in figure 1. The lines observed (Kelly and Palumbo 1973) were $\mathrm{Ne} \mathrm{v,} 2 \mathrm{p}^{2} \mathrm{~g}{ }^{3} \mathrm{P}-2 \mathrm{p} 3 \mathrm{~d}{ }^{3} \mathrm{P}^{\mathrm{o}}(142.52 \AA)$, Ne vi, $2 \mathrm{p}^{2} \mathrm{P}^{\mathrm{o}}-3 \mathrm{~d}{ }^{2} \mathrm{D}$ $(122.6 \AA)$, and $2 \mathrm{~s} 2 \mathrm{p}^{2}{ }^{4} \mathrm{P}-2 \mathrm{~s} 2 \mathrm{p}\left({ }^{3} \mathrm{P}^{\circ}\right) 3 \mathrm{~d}{ }^{4} \mathrm{D}^{\circ}(121 \cdot 1 \AA)$; Ne viI, $2 \mathrm{~s} 2 \mathrm{p}{ }^{1} \mathrm{P}^{\mathrm{o}}-2 \mathrm{~s} 3 \mathrm{~s}{ }^{1} \mathrm{~S}(127.7 \AA)$ and $2 \mathrm{~s} 2 \mathrm{p}{ }^{3} \mathrm{P}-2 \mathrm{~s} 3 \mathrm{~d}^{3} \mathrm{D}(106 \cdot 1 \AA)$; and Ne vin, $2 \mathrm{~s} \mathrm{~g}{ }^{2} \mathrm{~S}-3 \mathrm{p}{ }^{2} \mathrm{P}^{\circ}(88.1 \AA)$ and $2 \mathrm{p}^{2} \mathrm{P}^{\mathrm{o}}-3 \mathrm{~d}{ }^{2} \mathrm{D}$ $(98.2 \AA)$. For $\mathrm{Ne}$ vI the doublet and the quartet lines had the same time histories. For $\mathrm{Ne}$ VII the singlet and the triplet systems were found to have no detectable difference in the evolution of the lines. For $\mathrm{Ne}$ VIII, transitions to the ground state and to an excited state were analysed, and here both also had the same time development. Several weaker lines of these ionization stages were also observed, all of which demonstrated similar time evolution. Weak traces of $\mathrm{Ne}$ IV were also found.

The electron density was measured using the holographic interferometric set-up shown in figure 2 (plate) (Jahoda and Siemon 1972). In the analysis of these data, the plasma column was assumed to be $25 \mathrm{~cm}$ long. This is a good assumption since the holographic interferometric technique measures $N_{e} l$ where $l$ is the plasma length. The average ion velocity is of the order of $10^{7} \mathrm{~cm} \mathrm{~s}^{-1}$ and some of these ions and accompanying electrons will naturally flow down the field lines out the end of the tube. This may make the plasma length longer, but with a lower electron density at the end regions of the coil, while leaving the electron density at the coil midplane essentially unchanged. The total $N_{e} l$, however, should stay the same, and we can therefore accurately measure $N_{\mathrm{e}}$ at the coil midpoint by using $25 \mathrm{~cm}$ as the plasma length. A few typical holograms are also shown in figure 2 and the resulting effective electron density is plotted as a function of time in figure 3. Figure 4 shows the electron density as a function of radius for a few selected times after the initiation of the main bank discharge. As mentioned in the section on the theoretical model, this radial dependence of density is incorporated into the model in order to improve our experimental accuracy.

The electron temperature was measured by doing Thomson scattering at $90^{\circ}$ (DeSilva and Goldenbaum 1970). A 2 J ruby laser which emitted a light pulse of $20 \mathrm{~ns}$ duration was used as a light source. The resulting scattered light was scanned on a shot-to-shot basis with a $20 \mathrm{~cm}$ monochromator having a $40 \AA$ instrumental width. The early plasma formation reproducibility was monitored on a shot-to-shot basis with a broad-band filter at the $\mathrm{He}$ II $4686 \AA$ line. This broad-band filter also monitored the late-time continuum emission in the visible. The relative sensitivity of the detector was measured as a function of wavelength using a tungsten lamp and the resulting corrections applied to the scattered light profiles. The scattered light profiles were then fitted via least squares to a Gaussian. The electron temperatures, plotted against time, obtained by this procedure are shown in figure 5 . The 


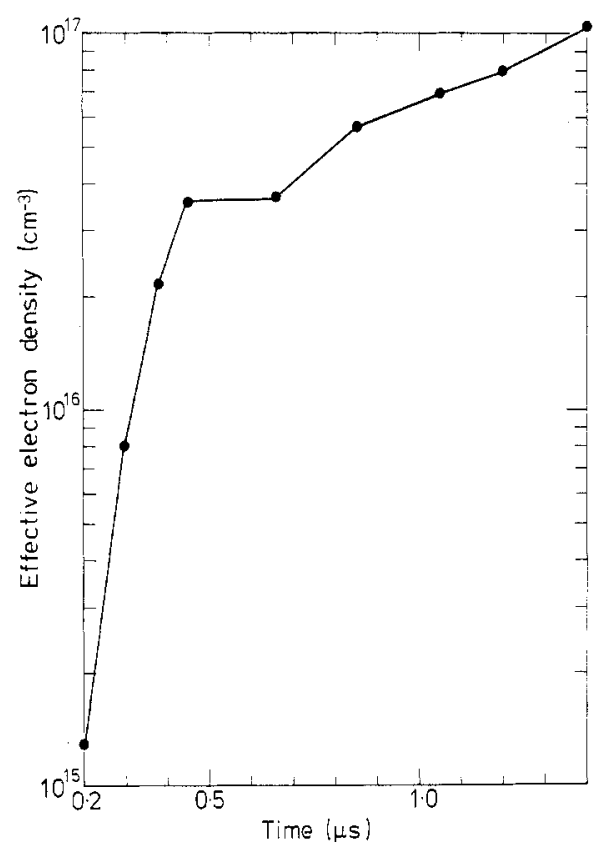

Figure 3. A plot, versus time, of the effective electron density, $N_{\text {eff }}=\frac{\int N_{\mathrm{e}}^{2}(r, t) \mathrm{d} r}{\int N_{\mathrm{e}}(r, t) \mathrm{d} r}$.

electron temperatures were measured at only one point in the plasma column and the assumption has been made that the temperature is constant as a function of radius within the column at the coil midplane. This assumption is reasonable since estimates of thermal transport in our plasma column yielded times which were faster

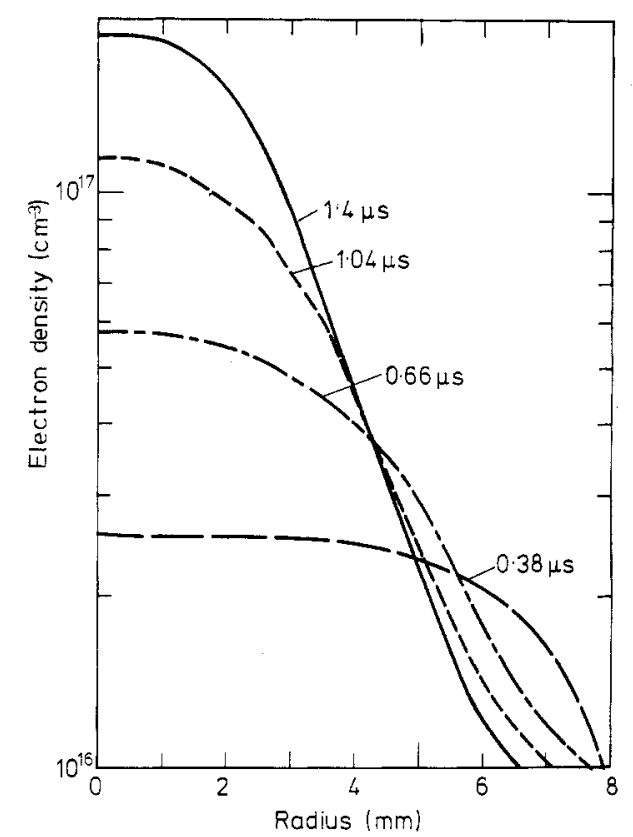

Figure 4. A plot of the electron density versus plasma radius at the coil midplane. 


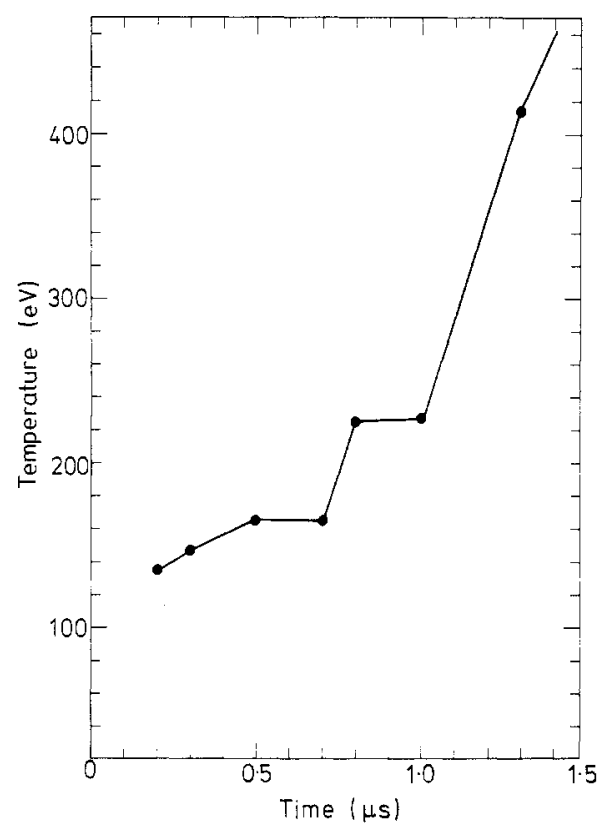

Figure 5. A plot of the electron temperature, obtained from $90^{\circ}$ Thomson scattering, versus time.

than the time scale for a significant change in temperature at the coil midplane. This at least assures us that the inhomogeneity in temperature was significantly less than the inhomogeneity in electron density.

\section{Theoretical model}

We model the plasma produced in our theta pinch by use of the set of rate equations (Düchs and Griem 1966):

$\frac{\mathrm{d} N_{z}}{\mathrm{~d} t}=N_{\mathrm{e}} N_{z-1} S_{z-1}-N_{\mathrm{e}} N_{z}\left(S_{z}+\alpha_{z-1}+N_{\mathrm{e}} \gamma_{z-1}\right)+N_{\mathrm{e}} N_{z+1}\left(\alpha_{z}+N_{\mathrm{e}} \gamma_{z}\right)$

where $N_{z}$ is the number density of the $z$ th ion, $N_{\mathrm{e}}$ the electron density, $S_{z}$ the ionization rate for the $z$ th ion, $\alpha_{z}$ the radiative recombination rate into the $z$ th ion and $\gamma_{z}$ the collisional recombination rate into the $z$ th ion.

We have estimated the various rates (Griem 1964, Summers 1974) which appear in these equations and have found for our extreme conditions, i.e. $N_{\mathrm{e}} \simeq 10^{17} \mathrm{~cm}^{-3}$ and $T_{\mathrm{e}} \simeq 200 \mathrm{eV}$, that the ionization rate dominates the radiative and collisional recombination rates by at least two orders of magnitude until $\mathrm{Ne}$ IX is considered. Since we do not model the emission from Ne Ix ions, we conclude that recombination can be ignored. However, as will be discussed in the concluding section, one of our measured ionization rates is almost an order of magnitude smaller than expected, thereby again raising the question of including the recombination rates. Thus equation (1) reduces to

$$
\mathrm{d} N_{z}(r, t)=N_{\mathrm{e}}\left(N_{z-1} S_{z-1}-N_{z} S_{z}\right) \mathrm{d} t
$$


where we assume that all quantities depend on the radius only. Our diagnostics of the plasma shows a variation of electron density with radius, as mentioned in the previous section. We have included this variation, assuming the radial distribution of all ions is the same as the distribution of electrons, i.e.,

$$
N_{z}(r, t)=N_{z}(0, t) \frac{N_{\mathrm{e}}(r, t)}{N_{\mathrm{e}}(0, t)} .
$$

This assumption, and the fact that we view the entire diameter of the plasma column side on, allows us to correct for plasma inhomogeneities by calculating the total number $N_{z}^{\mathrm{T}}$ of ions in our viewing volume. Since the axial dimension as viewed by the monochromator remains constant in time, there is no need to average over this dimension. The rate equations then become:

$$
\frac{\mathrm{d} N_{z}^{\mathrm{T}}(t)}{\mathrm{d} t}=\int \frac{\mathrm{d} N_{z}(r, t)}{\mathrm{d} t} \mathrm{~d} r=\left(S_{z-1} \int N_{\mathrm{e}}(r, t) N_{z-1}(r, t) \mathrm{d} r-S_{z} \int N_{\mathrm{e}}(r, t) N_{z}(r, t) \mathrm{d} r\right)
$$

where

$$
N_{z}^{\mathrm{T}}(t)=\int N_{z}(r, t) \mathrm{d} r=\frac{N_{z}(0, t)}{N_{\mathrm{e}}(0, t)} \int N_{\mathrm{e}}(r, t) \mathrm{d} r
$$

which gives

$$
\frac{d N_{z}^{\mathrm{T}}(t)}{\mathrm{d} t}=\frac{\int N_{\mathrm{e}}^{2}(r, t) \mathrm{d} r}{\int N_{\mathrm{e}}(r, t) \mathrm{d} r}\left[S_{z-1} N_{z-1}^{\mathrm{T}}(t)-S_{z} N_{z}^{\mathrm{T}}(t)\right]
$$

This set of coupled equations was integrated numerically to obtain $N_{z}^{\mathrm{T}}(t)$ using the experimentally determined time-dependent electron temperature and the timeand radius-dependent density. The ionization rates used in this calculation were the semi-empirical rates of Kunze (1971a). The recent calculations by Summers (1974) were not used because of the ease in putting the analytic form into our program. A comparison will be made later with this recent calculation to determine which ionization rates agree best with the experiment. The resulting total ground-state number density of the $z$ th ion, $N_{z}^{\mathrm{T}}(t)$, was then corrected for end losses in the plasma by scaling it like $\mathrm{d} N_{\mathrm{e}} / \mathrm{d} t$. We wanted to compare our measured time evolution of the line intensities with this model and to do this we assumed that every exciting collision leads to the emission of a photon. This assumption is valid if we restrict ourselves to observing only lines which originate from low-lying levels where the assumptions of coronal excitation equilibrium hold. We then obtain

$$
I_{m}^{n}(r, t) \propto N_{z}(r, t) N_{\mathrm{e}}(r, t) X_{0}^{m}
$$

where $I_{m}^{h}(r)$ is the intensity of light emitted by a transition from level $m$ to $n$ and $X_{0}^{m}$ is the collisional excitation rate from the ground state to level $m$. We can then integrate this expression over $r$ and obtain the total line emission

$$
I_{m}^{n}(t) \propto N_{z}^{\mathbf{T}}(t) X_{0}^{m} \frac{\int N_{\mathrm{e}}^{2}(r, t) \mathrm{d} r}{\int N_{\mathrm{e}}(r, t) \mathrm{d} r} .
$$

At our temperature conditions we are far above threshold for the excitation, and, 
for example, for Ne viII, a $70 \%$ increase in temperature at $100 \mathrm{eV}$ changes the excitation rates by only $5 \%$ (Kunze and Johnston 1971). Thus we assume that the temperature dependence, and hence the time dependence, of $X_{0}^{m}$ is weak and obtain

$$
I_{m}^{n}(t) \propto N_{z}^{\mathrm{T}}(t) \frac{\int N_{\mathrm{e}}^{2}(r, t) \mathrm{d} r}{\int N_{\mathrm{e}}(r, t) \mathrm{d} r} .
$$

\section{Results}

The measured and predicted line intensities of $\mathrm{Ne}$ VI, Ne vII and Ne vIII were each normalized to unity at maximum intensity and plotted in figure 6. Figure 6(a) compares the experimental intensities with the calculated intensities when the semi-empirical ionization rates of Kunze (1971a) were multiplied by unity. It is evident from the figure that the semi-empirical rates are much too large. Figure $6(b)$ shows the experimental intensities and the calculated curves obtained by adjusting the ionization rates to arrive at a best fit. The initial conditions chosen for the fitting program were such that the calculated time history of the $\mathrm{Ne} \mathrm{v}$ emission agreed with the

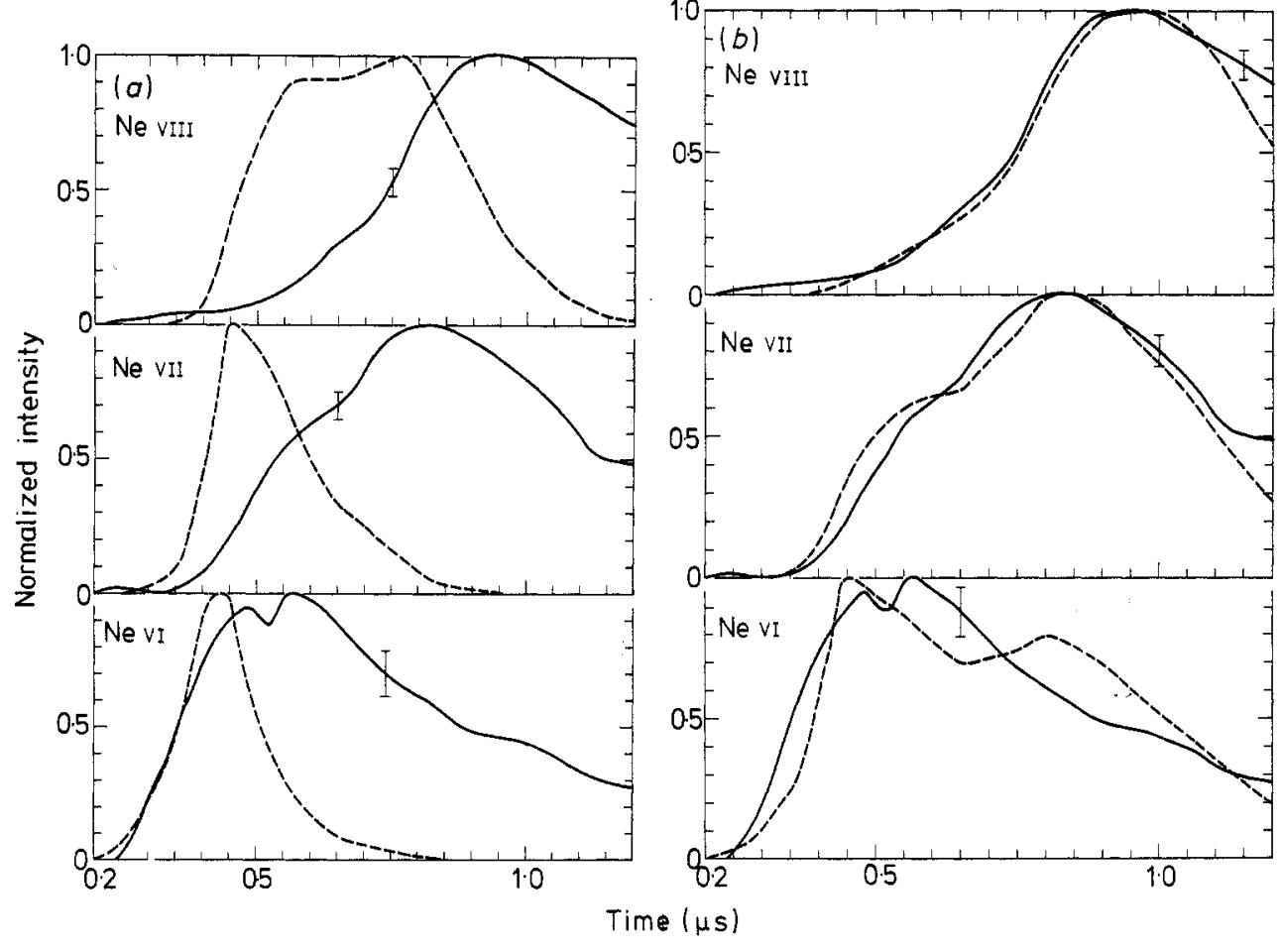

Figure 6. Comparisons of the experimentally observed light emissions (-) with the theoretically predicted light emissions (-- ) from Ne VI, VII and vII. The ionization rates used in the theoretical model were the semi-empirical rates of Kunze (1971a). In (a) these rates were unmodified whereas in $(b)$ each of the rates were multiplied by the constants given in table 1 . 
experimentally observed intensity. To obtain the best-fit curves, we had to multiply the semi-empirical ionization rates for the various ions by the factors shown in column two below.

Table 1

\begin{tabular}{lcl}
\hline Ne VI & $0.15 \pm 0.1$ & 1.64 \\
Ne vil & $0.4 \pm 0.2$ & 0.62 \\
Ne VIII & $0.7 \pm 0.25$ & 0.62 \\
\hline
\end{tabular}

The estimated errors reported reffect the minimum change in the factors for which we could distinguish a significant change in the best-fit curves $( \pm 0.05)$. They also include the estimated errors in our measurement of electron density $( \pm 10 \%)$, temperature $( \pm 10 \%)$ and the radial distribution of electron density $( \pm 5 \%)$, as well as the accuracy of the homogeneity correction calculation $( \pm 5 \%)$.

These results for Ne vil and vill are consistent with earlier work of Kunze (1972) who reported ionization rates about 50 to $60 \%$ lower than those given by his semiempirical formula (Kunze 1971a, Lotz 1967, 1968). It is important to note, however, that our measurements have a higher degree of accuracy and, unlike the earlier results, exclude the possibility of having a multiplicative factor of one. Of course, it is necessary to note that what we have measured is the total ionization rate. We have compared this to a calculated ionization rate from the ground state which has not included inner-shell ionization. Our model calculation has also not included internal atomic structure, i.e., excited states, metastable states, etc. It seems logical, however, that the addition of any other processes by which an atom could be ionized would only serve to raise the total calculated ionization rate and thereby make the discrepancy larger. One striking example of this is Ne vi where we have included ionization from the $2 \mathrm{p}$ level only. However, if we considered inner-shell ionization from $2 \mathrm{~s}$ as well, the predicted theoretical ionization rates would come out about three times larger, and the discrepancy for this ionization stage would be even worse.

As pointed out earlier in our model we have assumed a homogeneous temperature distribution in the plasma. This is an appropriate assumption for the late times but might be less valid in the early ion stage build-up. We excluded the $\mathrm{Ne} \mathrm{v}$ ionization rates from our comparison with predicted rates for this reason. The ionization rate found for Ne vi chiefly depends upon the decay of the Ne vi radiation, 0.5 to $1.0 \mu$ s, and therefore early inhomogeneities in the temperature distribution should not affect our results for this ion stage. Thus, we feel that the discrepancies found for the $\mathrm{Ne}$ vi ionization rates are real and not due to a large temperature inhomogeneity at early time.

To compare our results with the recent calculations of Summers (1974), we have entered in column three of table 1 the ratio of the ionization rates of Summers (1974) to the ionization rates calculated from Kunze's formulae at a density of $1 \times 10^{16} \mathrm{~cm}^{-3}$ and a temperature of $272.5 \mathrm{eV}$. This rather crude comparison shows that for Ne vil and VIII the Summers' rates agree with our experimentally determined rates within experimental and theoretical estimated accuracies. For Ne vi, however, the discrepancy with the experiment would be made much larger if the Summers' ionization rates were used. This seemingly larger discrepancy is due to the inclusion of inner-shell ionization by Summers which we have not included in our application of the Kunze semi-empirical formula to our problem. 
Finally, we wish to make the point that although the recently calculated ionization rates seem to be more accurate than those resulting from a previously derived semiempirical formula, there still may be instances, as with $\mathrm{Ne}$ VI, where the calculated values disagree badly with the measured quantities. Of course, this discrepancy could also be due to a much larger total recombination rate which we have ignored, but we feel a one order of magnitude error in the ionization rate is much more likely than a two orders of magnitude error in the recombination rate.

\section{Acknowledgment}

The authors wish to thank $\mathrm{M} \mathrm{McClannahan} \mathrm{for} \mathrm{his} \mathrm{technical} \mathrm{assistance} \mathrm{in} \mathrm{maintain-}$ ing the capacitor bank and aligning the optical apparatus.

\section{References}

Datla R U, Kunze H-J and Petrini D 1972 Phys. Rev. A 6 38-44

DeSilva A W and Goldenbaum G G 1970 Methods of Experimental Physics vol 9A (New York: Academic Press) chap 3

Düchs D and Griem H R 1966 Phys. Fluids 9 1099-109

Griem H R 1964 Plasma Spectroscopy (New York: McGraw-Hill) chap 6

Jahoda F C and Siemon R E 1972 Los Alamos Scientific Laboratory Report LA-5085-MS

Johnston W D III and Kunze H-J 1971 Phys. Rev. A 4 962-70

Kelly R L and Palumbo L J 1973 Naval Research Lab Report NRL-7599

Kunze H-J 1971a Phys. Rev. A 3 937-42

-1971b Phys. Rev. A 4 111-3

-1972 Space Sci. Rer. 13 565-83

Kunze H-J and Johnston W D III 1971 Phys. Rev. A 3 1384-93

Lotz W 1967 Astrophys. J. Suppl. 14207

_-1968 Institute für Plasma Physik, Garching bei München Report IPP 1/67

Summers H P 1974 Mon. Not. R. Astron. Soc. $169663-80$

Thomson D B, Jones L A, Bailey A G and Engleman R Jr 1976 J. Plasma Phys. to be published 